\title{
Electrophysiological Aspects of Human Sphincter Function
}

\author{
Thomas J. Ustach, Fabio Tobon, Terry Hambrecht, D. David Bass, and \\ MARVIN M. SchUSTER \\ From the Department of Medicine, Baltimore City Hospitals and Johns Hopkins \\ University School of Medicine, Baltimore, Maryland 21224
}

A B S T R A C T In order to investigate the electrophysiology of the human internal anal sphincter and two current concepts of sphincter function, simultaneous manometric and electrical recordings were made from circular smooth muscle of the internal anal sphincter in the resting state and during reflexly induced sphincter relaxation. Three groups were studied: seven normal subjects, 25 patients with functional bowel disease, and seven patients with external sphincter paralysis due to spinal cord lesions. In the resting state slow waves of alternating potential (basic electrical rhythm or BER) were recorded in all subjects. Two types of waves were present, a constant sinusoidal pattern or a spindleshaped pattern. Either pattern was consistent for a given individual. Frequency of BER in the internal sphincter was higher than that recorded in any other gastrointestinal muscle. Our findings indicate that the BER recorded from the internal anal sphincter originates in this muscle. This activity may represent a specialized feature of sphincteric muscle since BER cannot be recorded from isolated nonsphincteric circular muscle.

Reproduction of the two patterns of BER by an electronic model suggests that BER, as recorded by this technqiue, results from a summation of a number of electrically active cells in contact with the recording electrodes. Inhibition of BER occurred when sphincter relaxation was reflexly induced by rectal distension. Both inhibition of BER and degree of sphincter relaxation were proportional to the strength of rectal stimulation, suggesting that strength of stimulus determines the number of active cells which are inhibited.

The associations of high frequency of BER with high resting pressure, and of inhibition of BER with sphincter relaxation suggests that maintenance of sphincter tone is an active process that is governed by BER.

A partial report of this work was presented at the American Gastroenterological Association Meetings, 18 May 1968, Philadelphia, $\mathrm{Pa}$.

Received for publication 16 May 1969 and in revised form 15 August 1969.

\section{INTRODUCTION}

This study was undertaken to investigate electrical activity in the circular smooth muscle of the human internal anal sphincter and to evaluate two current hypotheses of sphincter function. Present concepts of electrophysiology of gastrointestinal smooth muscle are based on the observation that there are two types of electrical activity which can be recorded from the small intestine: slow sinusoidal waves of alternating potential called basic electrical rhythm (BER), and rapid spike activity. It is generally held that BER originates in longitudinal muscle since $B E R$ can be recorded in vitro in isolated longitudinal muscle but not in isolated circular muscle (1-8). BER is thought to set the stage for spike activity which is associated with circular muscle contraction (1,9-12). Our findings indicate that BER can originate from the internal anal sphincter which is composed of circular smooth muscle anatomically separate from longitudinal muscle.

One of two current concepts explaining the high resting pressure (which we shall refer to as "tone") within the anal sphincter proposes that the internal sphincter is held closed by an active process and that sphincter relaxation results from an inhibition of this state (13). According to the second theory the sphincter, when closed, is in an inactive state, but distension from within initiates active resistance (14). Our findings obtained by recording simultaneous electrical and manometric activity within the sphincter support the first hypothesis.

The high frequency of BER which was associated with a high resting pressure, as well as inhibition of BER which accompanied sphincter relaxation, suggests that BER may be related to tone.

\section{METHODS}

Studies were performed on 39 subjects: seven normal volunteers, 25 patients with functional bowel disorder, and seven patients with spinal cord lesions. Normal volunteers ranged in age from 31 to $74 \mathrm{yr}$ (average of $45 \mathrm{yr}$ ). The patients with functional bowel disorders ranged in age from 18 months to 


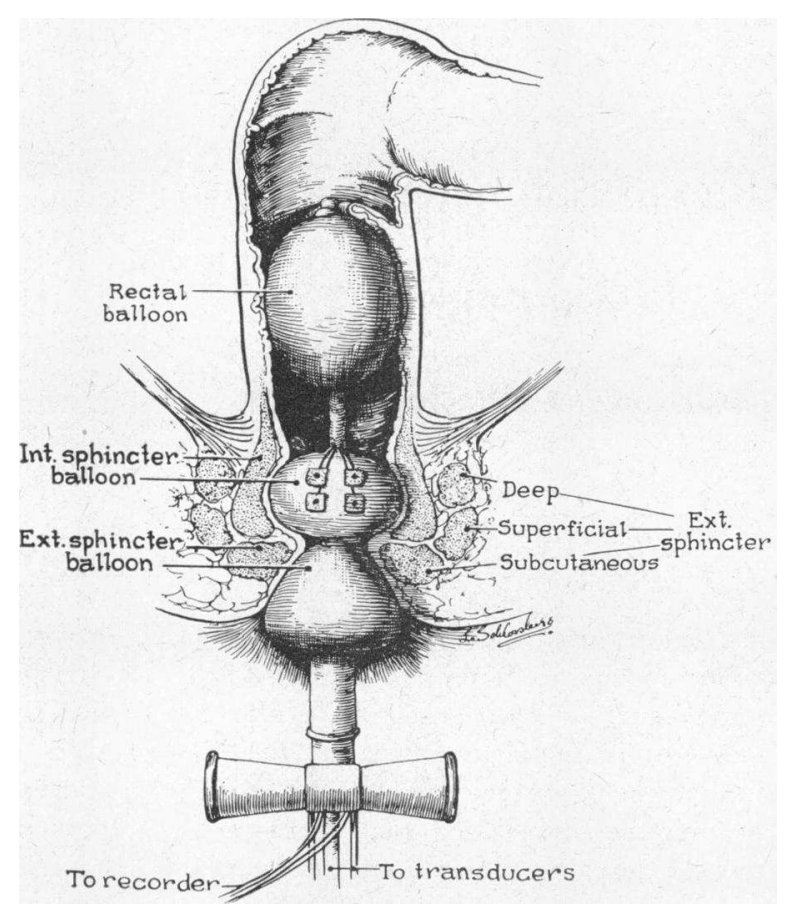

FIGURE 1 Schematic diagram of recording technique. The anatomical relationship of the pressure-sensing device in the anal canal is shown. The flat surface electrodes used for electrical recordings are cemented onto the balloon which is surrounded by the internal sphincter.

$80 \mathrm{yr}$ (average of $30 \mathrm{yr}$ ). These patients had vague abdominal complaints and constipation, or constipation alternating with diarrhea. At the time of the study constipation was the presenting problem in 20 patients and diarrhea in the remaining five patients. The patients with spinal cord lesions ranged in age from $4 \mathrm{yr}$ to $63 \mathrm{yr}$ (average of $42 \mathrm{yr}$ ). The lesions, located from $\mathrm{T}-12$ to $\mathrm{S}-2$, resulted in external sphincter paralysis and symptoms of constipation and incontinence.

Pressure recordings were obtained from a double balloon device tied around a hollow steel cylinder to form two separate compartments (15). This device was inserted into the anal canal so that the internal balloon, when inflated with $10 \mathrm{cc}$ of air, was surrounded by the internal anal sphincter and the external balloon when similarly inflated was encircled by the superficial bundle of the external sphincter (Fig. 1). A third balloon, inserted through the hollow core of the recording device, was used to initiate reflex sphincteric responses by transient rectal distension (for $2 \mathrm{sec}$ ) with $10-50 \mathrm{cc}$ of air, progressing in $10 \mathrm{cc}$ increments. When filled with $50 \mathrm{cc}$ of air the rectal balloon distended laterally, was $5 \mathrm{~cm}$ long, and remained $4 \mathrm{~cm}$ above the internal sphincter balloon. Respirations were also monitored by this balloon. Each of the balloons communicated by polyethylene tubing with pressure transducers. ${ }^{1}$ Pressure tracings were displayed on three channels of a four channel direct writing recorder. ${ }^{2}$

\footnotetext{
${ }^{1}$ Sanborn model 267B.

Sanborn model 964 (frequency response: D.C. to 125 Hz.).
}

Electrical recordings were obtained from the internal sphincter area by electrodes cemented to the surface of the internal sphincter balloon. The electrodes were made by removing a centimeter of insulation from $0.2 \mathrm{~mm}$ silverplated copper wire (Tensolite No. 32 gauge, Teflon insulated) which had a resistance of $0.408 \mathrm{ohms} / \mathrm{m}$. This was inserted into a small patch of latex leaving a $1.0 \mathrm{~mm}$ length of stripped wire as an electrode. The patch was then fastened to the internal sphincter bolloon with waterproof insulating cement (see Fig. 1). These electrodes, arranged in pairs $1 \mathrm{~cm}$ apart, communicated with an input adapter ${ }^{3}$ and a low level preamplifier." Bipolar techniques were used because, when compared, bipolar tracings were similar to monopolar tracings, contained less outside interference, and eliminated electrocardiographic artefacts. Electrical tracings were displayed on the fourth channel of the direct writing recorder.

In order to differentiate electrical activity of smooth muscle from that of striated muscle, electromyography was recorded from the striated muscle of the external anal sphincter (superficial bundle) by a platinum concentric needle electrode. The electromyographic recording was displayed on an oscilloscope ${ }^{5}$ and a loudspeaker. ${ }^{5}$

Recordings were made from the internal sphincter for 3 min in the resting state and then during sphincter relaxations induced by rectal distension. Both pressure and electrical recordings were analyzed for amplitude, frequency, and wave form. A recognizable deviation from the isoelectric line of $80 \mu \mathrm{v}$ ( $2 \mathrm{~mm}$ with our usual recording sensitivity) was accepted as an electrical wave. An electrical cycle was defined as a wave which began at the isoelectric line, became displaced above this line and then below it, and then returned to the isoelectric line. Correlation of electrical activity and pressure changes were made at paper speeds of $1 \mathrm{~mm}, 2.5 \mathrm{~mm}$, and $5 \mathrm{~mm} / \mathrm{sec}$.

\section{RESULTS}

Resting state. A basic electrical rhythm (BER) consisting of slow waves of alternating potential was recorded from the internal sphincter in all subjects. Two types of electrical activity were present. A constant sinusoidal pattern (Fig. 2) was found in 18 subjects and a spindle or diamond-shaped pattern (Fig. 3) in 22 subjects. Each pattern appeared in approximately half of subjects in each of the three study groups (Table I). A given pattern was typical for a given subject since it remained unchanged throughout the entire study and was reproducible in five subjects on repeat study. BER was independent of respiratory excursions since it differed from respiration in frequency and was not altered by breath holding (Fig. 4). In no instance was spike activity recorded.

In normal subjects the average frequency of BER was 17 cycles $/ \mathrm{min}$. The average frequency for the other two groups having bowel complaints was somewhat lower (Fig. 5). Whereas the frequency of BER was the same on repeat studies in a given subject, voltage was

\footnotetext{
${ }^{3}$ Sanborn model 56A.

- Sanborn model 350-3A.

${ }^{5}$ Disa model 13B09.
} 


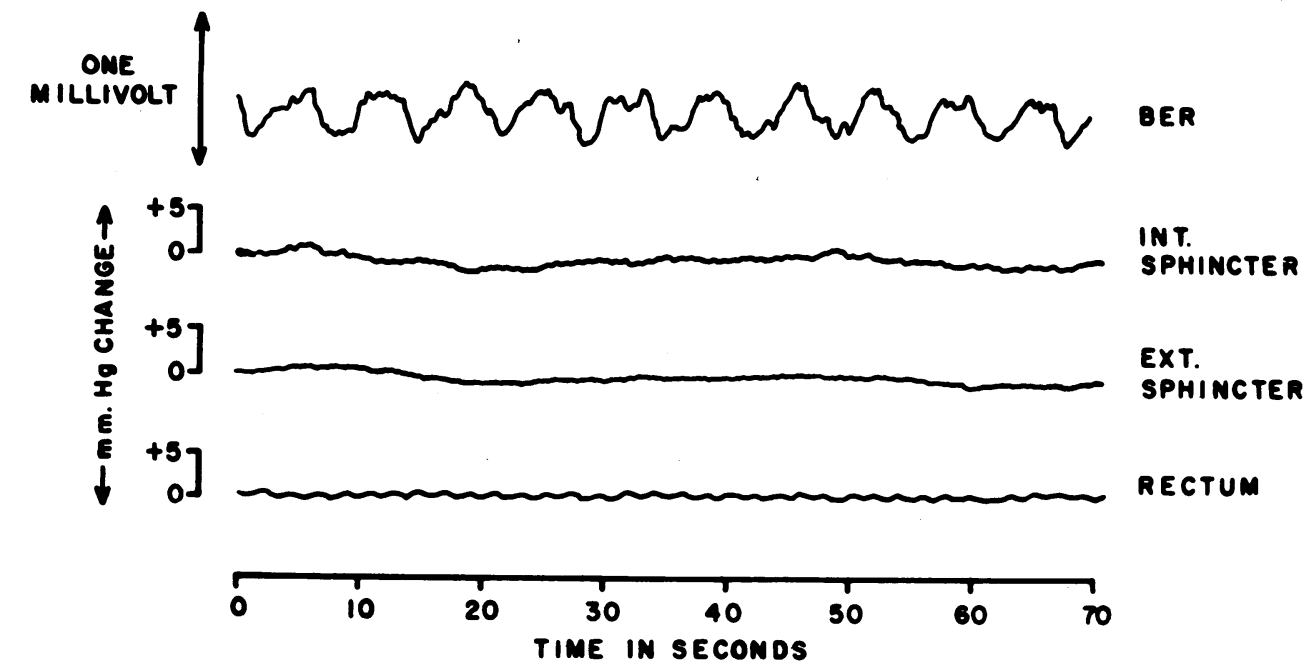

FIGURE 2 Constant sinusoidal BER in the resting state. A sinusoidal basic electrical rhythm (BER) is recorded from the internal sphincter at a time that no pressure changes are occurring in this sphincter. Upper tracing is the bipolar electrical recording from the internal anal sphincter and the lower three tracings are pressure recordings from the balloons placed in the designated areas. In this and subsequent figures resting pressure in each balloon is assigned a zero value on the vertical axis. Increases above this level (contraction) are designated as positive, and decreases (relaxation) as negative.

not. Voltage in all subjects ranged from 200 to $600 \mu \mathrm{v}$. Neither voltage nor frequency correlated with age or sex distribution.

Sphincteric responses. Rectal distension produced relaxation of the internal anal sphincter. Inhibition of electrical activity (BER) was associated with internal sphincter relaxation and appeared with the onset of relaxation. Both the inhibition of electrical activity and the degree of sphincter relaxation were related to the volume of air used for the rectal distension (Fig. 6). When $50 \mathrm{cc}$ of air was used for rectal distension, inhibition of electrical activity (Fig. 7) occurred in $73 \%$ of distensions; when $20 \mathrm{cc}$ of air was used, inhibition accompanied only $5 \%$ of distensions (Fig. 8). No



FIGURE 3 Spindle shaped BER in the resting state. A crescendo-decrescendo pattern of BER is present in the internal sphincter (upper tracing) and is not associated with pressure changes. Patient movement (at the arrow) produces pressure increases in the internal and external sphincter balloons but does not alter the electrical activity. Electrical frequency was calculated as the number of cycles per minute between two areas of electrical inhibition. 

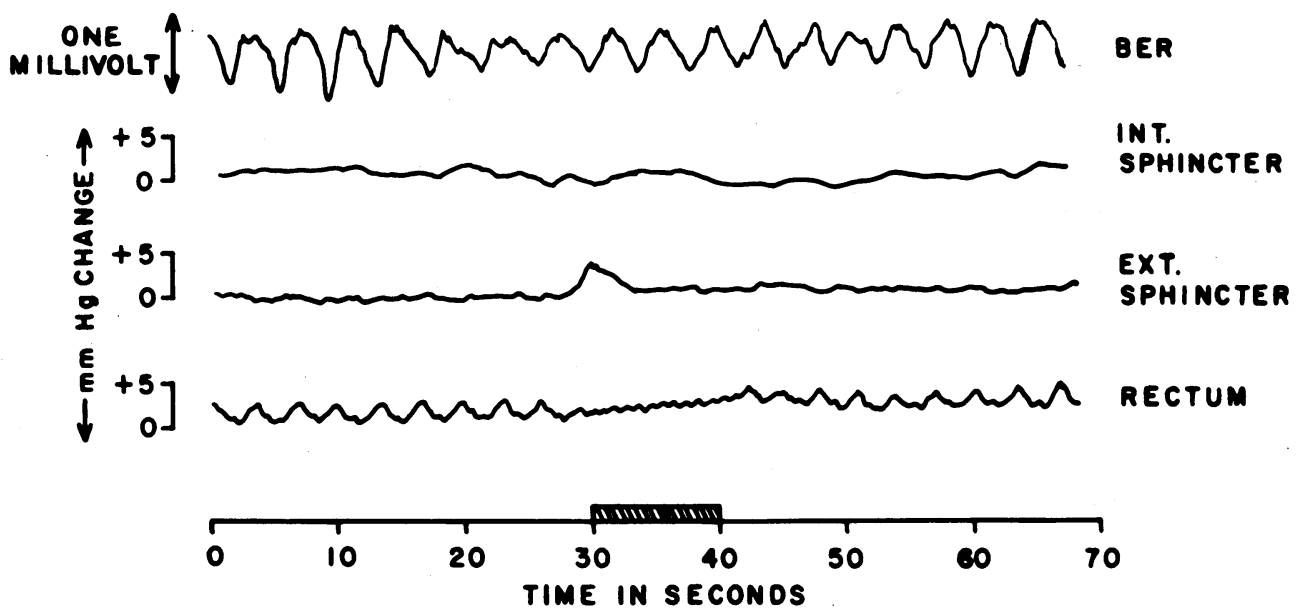

FIGURE 4 Demonstration that BER is independent of respiratory movements. $10 \mathrm{sec}$ of breath holding (indicated by the hatched bar) eliminated respiratory excursions in the rectal balloon but did not alter the electrical activity (BER).

change in electrical activity occurred with distending volumes of $15 \mathrm{cc}$ or less. When the sphincter itself was distended by inflating the internal sphincter balloon with an additional $10 \mathrm{cc}$ of air, there was no change in BER.

In the normal group and the group with functional bowel disorders rapid bursts of spike potentials were recorded from the external sphincter during voluntary or reflexly induced active contraction (reflex contraction of the external sphincter was stimulated by balloon distension of the rectum or by perianal scratch). When the external sphincter was in a resting state an occasional spike was seen on the oscilloscope. However in the group with spinal cord lesions no electrical activity was recorded from the external sphincter during the resting state or with rectal distension.

\section{DISCUSSION}

Origin of sphincteric BER. These studies demonstrating that BER can be recorded from circular smooth muscle in vivo are in contrast with in vitro studies of nonsphincteric circular smooth muscle from which no BER could be recorded $(1,2,16)$. Since our original report of these studies at the American Gastroentero-

TABLE I

Distribution of the Two Patterns of BER

\begin{tabular}{lccc}
\hline \multicolumn{1}{c}{ Subjects } & No. & $\begin{array}{c}\text { Constant } \\
\text { pattern }\end{array}$ & $\begin{array}{c}\text { Spindle } \\
\text { pattern }\end{array}$ \\
\hline & & $\%$ & $\%$ \\
Normal & 7 & 43 & 57 \\
Functional & 25 & 52 & 48 \\
Neurological & 7 & 43 & 57 \\
\hline
\end{tabular}

logical Association meetings in Philadelphia 18 May 1968, similar recordings of BER have been recorded from the internal anal sphincter using a different technique (17). The following is evidence that electrical

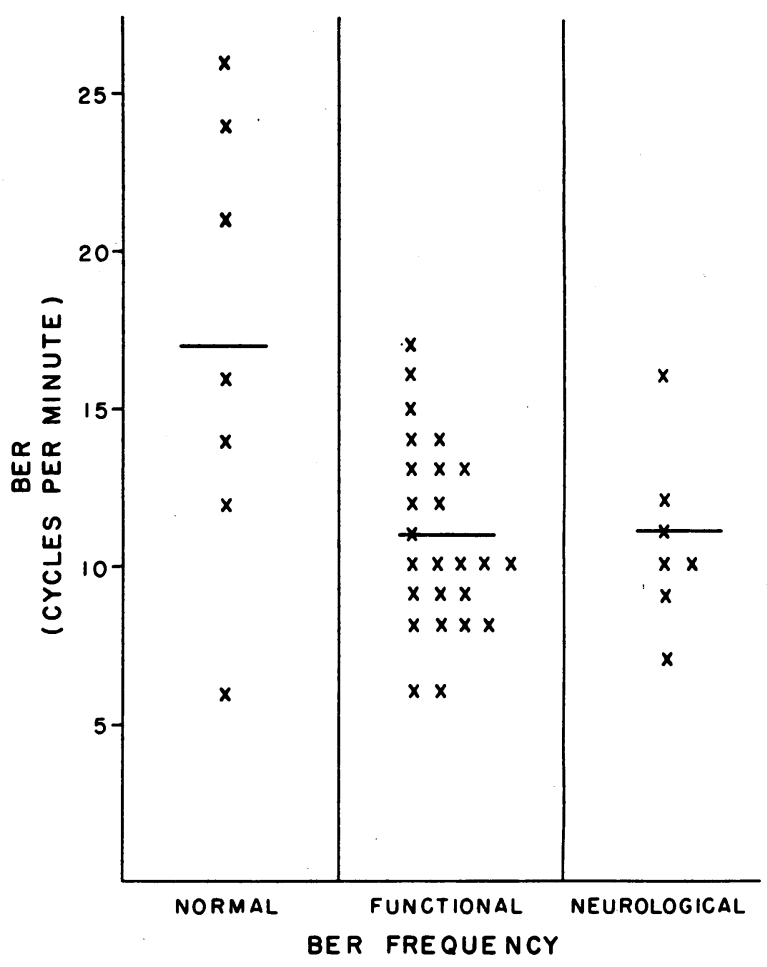

FIGURE 5 Distribution of BER frequency. Horizontal bar indicates the average frequency of BER in each group. Although the means for the functional and neurological groups differ from the normal, there is overlap among patients and the differences are not statistically significant (Student's $t$ test). 


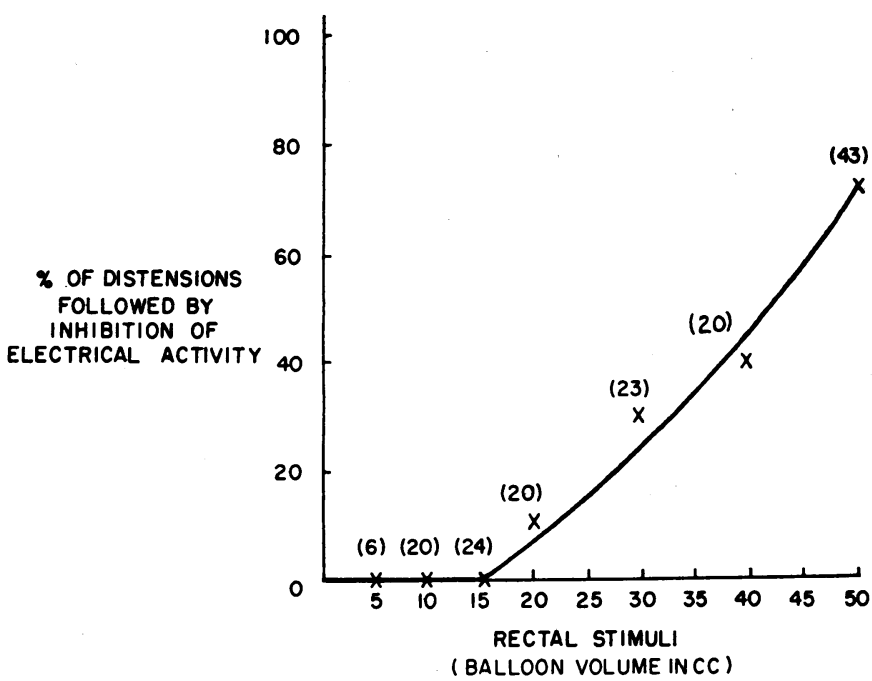

FIGURE 6 Inhibition of electrical activity with sphincter relaxation. Figures in parentheses represent total number of distensions at each designated volume. There is a direct correlation between strength of stimulus and inhibition of electrical activity.

activity truly originates in the circular smooth muscle of the internal anal sphincter. (a) This muscle is anatomically separate from and devoid of longitudinal muscle. Longitudinal muscle terminates above the internal sphincter and only sparse fibroelastic strands, which are extensions of longitudinal muscle, traverse the sphincter $(18,19)$. (b) Electrical activity is transmitted from longitudinal to circular muscle for extremely short distances $(1-2 \mathrm{~mm})(7)$ and this conduction is through connecting muscle fibrils (20). These two considerations
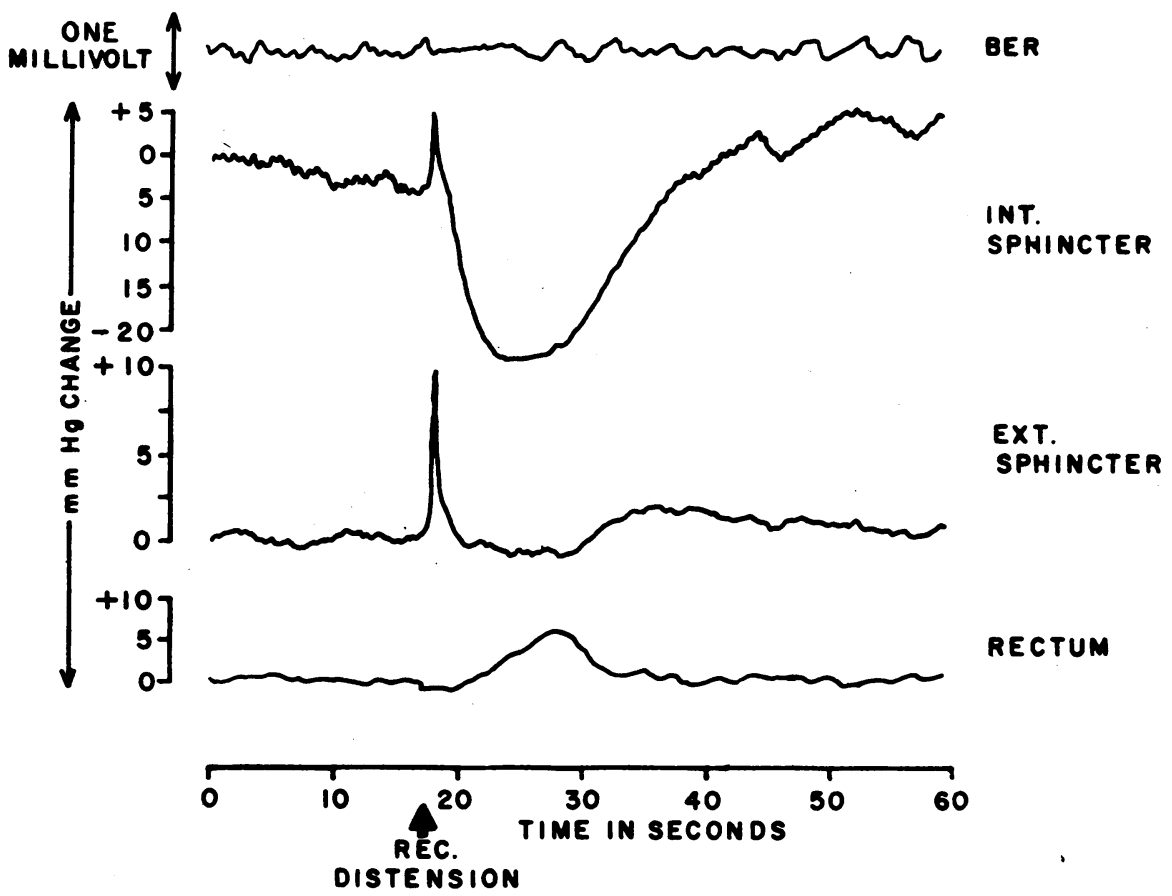

FIGURE 7 Inhibition of BER during internal sphincter relaxation. Inhibition of BER (upper tracing) accompanies the down slope of internal sphincter relaxation (second tracing) produced by rectal distension (arrow) with $50 \mathrm{cc}$ of air. BER returns at the same time that sphincter tone returns. 
argue against the transmission of electrical activity from longitudinal muscle which terminates above the sphincter. (c) BER in the internal sphincter is not transmitted from the adjoining rectal area since the BER in the rectum has a frequency of $3-6$ cycles $/ \mathrm{min}$ (21) while that in the sphincter has a frequency of 6-26 cycles/min. Elsewhere in the gastrointestinal tract frequency of BER slows progressively in a cephalo-caudad direction from duodenum to colon $(2,8,9,16,22,23)$. Average frequency of BER in the internal sphincter is not only faster than that of adjacent rectum, but faster than any other area of the human gastrointestinal tract so far studied $(12,24)$. (d) Two adjacent areas (in this case rectum and sphincter) can have different patterns of electrical activity without one area affecting the basic pattern of the other (25). (e) BER is not transmitted from the striated muscle of the external sphincter since electrical activity from this and other striated muscle does not consist of BER, but instead is characterized by very rapid bursts of spike potentials which occur only during muscle contraction (26). Furthermore BER still could be recorded from the internal sphincter in the group of patients with external sphincter paralysis due to spinal cord lesions. These subjects had a normal internal anal sphincter response to rectal distension but no activity, electrical or manometric, could be detected in the external anal sphincter.
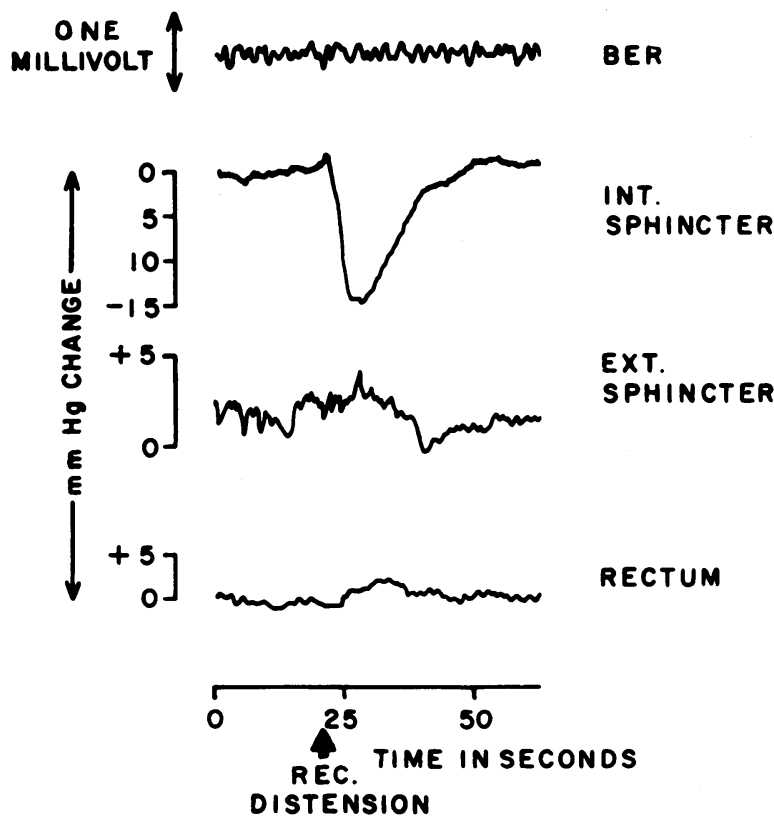

FIGURE 8 Internal sphincter relaxation without change in $\mathrm{BER}$. Internal sphincter relaxation (second tracing) has no effect on BER (upper tracing) when small volumes (20 cc) are used for rectal distension.

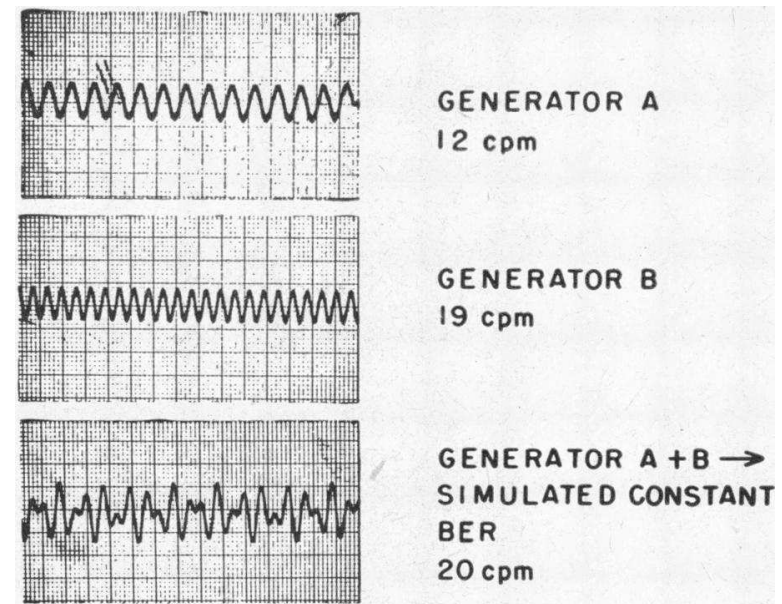

FIGURE 9 Artificially simulated BER (constant pattern). Constant pattern (lower tracing) similar to that recorded in vivo is produced by the summated activity of two generators ( $\mathrm{A}$ and $\mathrm{B}$ ) with frequencies that are out of phase but do not cancel each other.

These findings indicate that the BER recorded from the internal anal sphincter originates in this muscle. This activity may represent a specialized feature of sphincteric muscle since BER cannot be recorded from isolated nonsphincteric circular smooth muscle in vi ro.

Electrophysiological features relating to BER. The origin of the two types of BER as well as their differences may be explained electrophysiologically. In the intestine $(20)$, as in the brain and heart, groups of cells act as electrical generators with each generator discharging at its own frequency and voltage. The con-

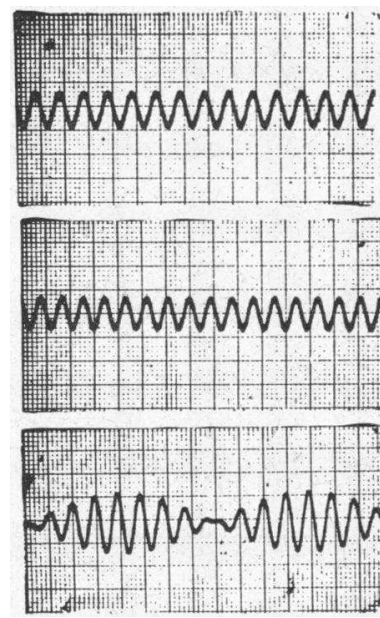

GENERATOR A $12 \mathrm{cPm}$

GENERATOR B $13.5 \mathrm{cpm}$

GENERATOR $A+B \rightarrow$ SIMULATED BER $12.1 \mathrm{cpm}$
Figure 10 Artificially simulated BER (spindle pattern). Spindle pattern (lower tracing) is produced by the summated activity of two generators (A and B) with frequencies that are out of phase in such a manner that they alternately augment and cancel each other. 


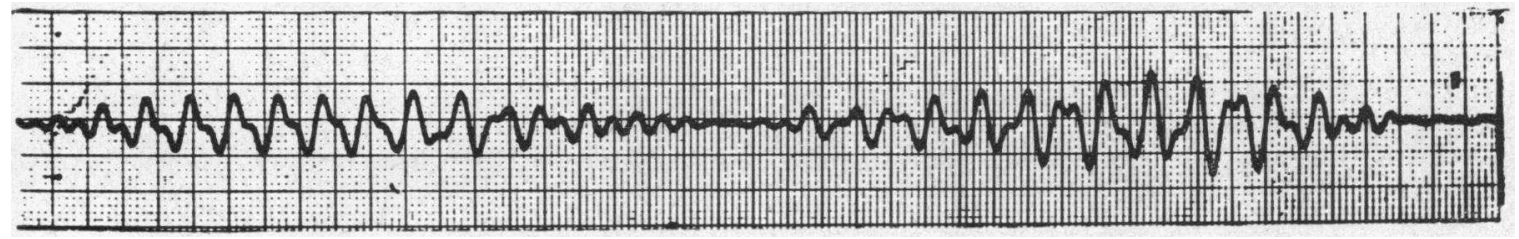

FigURE 11 Artificially simulated BER (spindle pattern similar to in vivo recording). Spindle pattern is produced by two generators ( $A$ and $B$ ) discharging as in Fig. 10, but with random changes in voltage. This same pattern may also result from an indefinite number of generators each firing at its own frequency and voltage.

stant pattern would be expected to result from electrical generators discharging at frequencies in synchrony or at frequencies which, though not synchronous, do not cancel each other. The spindle pattern would result from frequencies which are out of phase in such a manner that they do intermittently cancel each other. These conditions can be reproduced artificially by a model using two or more electronic generators ${ }^{6}$ which produce electrical activity at different frequencies. Fig. 9 shows how the constant pattern can be produced artificially by two generators even if they are producing waves' of different frequencies. Fig. 10 shows how an idealized spindle pattern can result from two generators having frequencies that alternately summate and cancel. Fig. 11 shows how a spindle pattern very much like that recorded in our subjects can be produced by two generators discharging as in Fig. 10 but with the additional feature of random changes in voltage. Although the above wave forms were produced using only two generators, it can be seen that the same results would be obtained from a large number of generators, a situation which resembles the in vivo circumstances.

Relation of BER to sphincteric relaxation. When strong stimuli were used for inducing sphincteric relaxat on BER was interrupted with greater frequency than when weaker stimuli were used. This correlation between volume of rectal distension, depth of sphincteric relaxation, and inhibition of BER suggests that inhibition is recorded only when stimulation is sufficiently strong to affect a relatively large number of active centers in the area of the recording electrodes. Since the electrodes measured $0.25 \mathrm{~mm}^{2}$ they recorded from a large number of active cells, not all of which were inhibited by rectal distension. Therefore not all induced relaxations would be expected to demonstrate inhibition of electrical activity.

Inhibition of BER was not due to movement of sphincter muscle away from the electrodes, since movement artefact has an entirely different pattern, consisting of rapid deflections of high amplitude. Loss of contact produced by deflating the internal sphincter balloon caused similar sharp excursions, usually with 60

\footnotetext{
- Wavetek Function Generator 110B.
}

cycle interference. Neither type of movement produced the straight line seen with sphincter relaxation. Recent studies (27) have shown that moving electrodes away from muscle or reducing the pressure of electrode contact results not in inhibition, but in a change of configuration of the wave form.

Relation of BER to sphincter tone. The following is evidence that BER is related to tone. (a) BER is the only type of electrical activity recorded from this sphincter. Spike activity is absent even though our apparatus is capable of recording spike potentials as demonstrated by passing a Miller-Abbott balloon, with similar electrodes attached, into the duodenum of healthy volunteers. Under these circumstances the expected spike potentials were recorded with intestinal contraction. (b) The frequency of BER in the internal sphincter is independent of neighboring muscle and does not follow the general trend of gradual slowing of BER frequency in a cephalocaudad direction $(3,8,10,16,20)$. The sphincter has a much higher resting pressure ("tone") than adjacent colon and also a much faster frequency of $\operatorname{BER}(21,24)$. (c) When atony of intestinal muscle is produced by general anesthesia, the frequency of BER is much slower than in the unanesthetized state (8).

The inhibition of BER during sphincter relaxation tends to support the concept that the sphincter is normally in a state of tonic contraction and that relaxation results from inhibition of this state. The electrical characteristics demonstrated by the present study suggest that in this functionally spec:alized area BER is related to the maintenance of tone.

\section{ACKNOWLEDGMENTS}

The authors wish to express their gratitude to Dr. Thomas $R$. Hendrix for his helpful suggestions in the preparation of this manuscript.

This work was supported by grant No. AM 07862 from the National Institutes of Health.

\section{REFERENCES}

1. Prosser, C. L. 1965. Symposium on gastrointestinal smooth muscle. Introduction to symposium. Gastroenterology. 49: 389.

2. Farrar, J. T., and A. M. Zfass. 1967. Small intestinal motility. Gastroenterology. 52: 1019. 
3. Bortoff, A. 1961. Electrical activity of intestine recorded with pressure electrodes. Amer. J. Physiol. 201: 209.

4. Bortoff, A. 1961. Slow potential variations of small intestine. Amer. J. Physiol. 201: 203.

5. Bozler, E. 1938. The action potentials of visceral smooth muscle. Amer. J. Physiol. 124: 502.

6. Daniel, E. E., D. R. Carlow, B. T. Wachter, W. H. Sutherland, and A. Bogoch. 1959. Electrical activity of the small intestine. Gastroenterology. 37: 268.

7. Papasova, M. P., T. Nagai, and C. L. Prosser. 1968. Two component slow waves in smooth muscle of cat stomach. Amer. J. Physiol. 214: 695.

8. Daniel, E. E., A. J. Honour, and A. Bogoch. 1960. Electrical activity of the longitudinal muscle of dog small intestine studied in vivo using microelectrodes. Amer. J. Physiol. 198: 113.

9. Daniel, E. E., and K. M. Chapman. 1963. Electrical activity of the gastrointestinal tract as an indication of mechanical activity. Amer. J. Dig. Dis. 8: 54.

10. Bass, P., C. F. Code, and E. H. Lambert. 1961. Motor and electric activity of the duodenum. Amer. J. Physiol. 201: 287.

11. Bass, P., and J. N. Wiley. 1965. Electrical and extraluminal contractile-force activity of the duodenum of the dog. Amer. J. Dig. Dis. 10: 183.

12. Christensen, J., H. P. Schedl, and J. A. Clifton. 1964. The basic electrical rhythm of the duodenum in normal human subjects and in patients with thyroid disease. J. Clin. Invest. 43: 1659.

13. Fleshler, B., T. R. Hendrix, P. Kramer, and F. L. Ingelfinger. 1958. Resistance and reflux function of the lower esophageal sphincter. J. Appl. Physiol. 12: 339.

14. Harris, L. D., and C. E. Pope. 1964. "Squeeze" vs. resistance: an evaluation of the mechanism of sphincter competence. J. Clin. Invest. 43: 2272.

15. Schuster, M. M., P. Hookman, T. R. Hendrix, and A. I. Mendeloff. 1955. Simultaneous manometric re- cording of internal and external anal sphincteric reflexes. Bull. Johns Hopkins Hosp. 116: 79.

16. Bass, P. 1965. Electrical activity of smooth muscle of the gastrointestinal tract. Gastroenterology. 49: 391.

17. Wankling, W. J., B. H. Brown, C. D. Collins, and H. L. Duthie. 1968. Basal electrical activity in the anal canal in man. Gut. 9: 457.

18. Stonesifer, G. L., Jr., G. P. Murphy, and C. R. Lombardo. 1960. The anatomy of the anorectum. Amer. J. Surg. 100: 66 .

19. Wilde, F. R. 1949. The anal intermuscular septum. Brit. J. Surg. 36: 279.

20. Bortoff, A. 1965. Electrical transmission of slow waves from longitudinal to circular intestinal muscle. Amer. J. Physiol. 209: 1254.

21. Hellemans, J., G. Vantrappen, P. Valenbois, J. Janssens, and J. Vandenbroucke. 1968. Electrical activity of striated and smooth muscle of the esophagus: discussion. Amer. J. Dig. Dis. 13: 320.

22. Bloom, A. A., P. LoPresti, and J. T. Farrar. 1968. Motility of the intact human colon. Gastroenterology. 54: 232.

23. Ludwick, J. R., J. N. Wiley, and P. Bass. 1968. Extraluminal contractile force and electrical activity of reversed canine duodenum. Gastroenterology. 54: 41.

24. Couturier, D., C. Rozé, M.-H. Couturier-Turpin, and C. Debray. 1968. Electromyographie per electrode unipolaire du côlon humain in situ. C. R. Acad. Sci. Paris. Ser. D. $264: 352$.

25. Bortoff, A., and R. S. Davis. 1968. Myogenic transmission of antral slow waves across the gastroduodenal junction in situ. Amer. J. Physiol. 215: 889.

26. Alva, J., A. I. Mendeloff, and M. M. Schuster. 1967. Reflex and electromyographic abnormalities associated with fecal incontinence. Gastroenterology. 53: 101.

27. Kobayashi, M., C. L. Prosser, and T. Nagai. 1967. Electrical properties of intestinal muscle as measured intracellularly and extracellularly. Amer. J. Physiol. 213: 275. 